\title{
NEW KERNELS IN QUANTUM GRAVITY
}

\author{
Giampiero Esposito \\ Istituto Nazionale di Fisica Nucleare, Sezione di Napoli, Mostra d'Oltremare Padiglione \\ 20, 80125 Napoli, Italy \\ Università di Napoli Federico II, Dipartimento di Scienze Fisiche, Complesso Universitario \\ di Monte S. Angelo, Via Cintia, Edificio G, 80126 Napoli, Italy
}

\begin{abstract}
Recent work in the literature has proposed the use of non-local boundary conditions in Euclidean quantum gravity. The present paper studies first a more general form of such a scheme for bosonic gauge theories, by adding to the boundary operator for mixed boundary conditions of local nature a $2 \times 2$ matrix of pseudo-differential operators with pseudo-homogeneous kernels. The request of invariance of such boundary conditions under infinitesimal gauge transformations leads to non-local boundary conditions on ghost fields. In Euclidean quantum gravity, an alternative scheme is proposed, where non-local boundary conditions and the request of their complete gauge invariance are sufficient to lead to gauge-field and ghost operators of pseudo-differential nature. The resulting boundary conditions have a Dirichlet and a pseudo-differential sector, and are pure Dirichlet for the ghost. This approach is eventually extended to Euclidean Maxwell theory.
\end{abstract}




\section{Introduction}

Recent efforts in the literature have led to an elegant and useful characterization of gaugeinvariant boundary conditions for linearized quantum gravity and other fundamental field theories. The basic elements of the Euclidean formulation are as follows [1].

A vector bundle $V$ with connection $\nabla$ is given over an $m$-dimensional Riemannian manifold $(M, g)$. Physical fields are the smooth sections of $V$, and a part of the boundary conditions is obtained by applying a projector $\Pi$ to the field $\varphi$ :

$$
[\Pi \varphi]_{\partial M}=0
$$

where $\partial M$ is the smooth boundary of $M$. Infinitesimal gauge transformations map $\varphi$ into

$$
{ }^{\varepsilon} \varphi=\varphi+R \varepsilon
$$

where $R$ is the generator and $\varepsilon$ is a 'gauge function' (for gravity, $\varepsilon$ is a 1-form; for YangMills, $\varepsilon$ is a smooth function on $M)$. Remaining boundary conditions are obtained by requiring that the action on $\varphi$ of the adjoint generator $\bar{R}$ should vanish on the boundary, i.e.

$$
[\bar{R} \varphi]_{\partial M}=0
$$

where $\bar{R} \equiv \gamma^{-1} R^{\dagger} E, E$ and $\gamma$ being the Hermitian metrics on the vector bundles of physical fields and 'gauge functions', respectively. Equation (1.3) is equivalent to setting to zero on the boundary the gauge-averaging functional (recall that Gaussian averages over gauge functionals are necessary to obtain an invertible gauge-field operator in the one-loop quantum theory [1]). The boundary conditions (1.1) and (1.3) can be re-expressed in the convenient form [1]

$$
\left(\begin{array}{cc}
\Pi & 0 \\
\Lambda & I-\Pi
\end{array}\right)\left(\begin{array}{c}
{[\varphi]_{\partial M}} \\
{[\varphi ; N]_{\partial M}}
\end{array}\right)=0
$$

where $\Lambda$ is a first-order differential operator on the boundary:

$$
\Lambda \equiv(I-\Pi)\left[\frac{1}{2}\left(\Gamma^{i} \widehat{\nabla}_{i}+\widehat{\nabla}_{i} \Gamma^{i}\right)+S\right](I-\Pi)
$$


$\Gamma^{i}$ being a set of vector fields on $\partial M, \widehat{\nabla}$ being the induced connection on $\partial M$, and $S$ being an endomorphism (e.g. a term proportional to the trace of the extrinsic-curvature tensor of the boundary). Of course, $; N \equiv N^{a} \nabla_{a}$ in Eq. (1.4) is the standard notation for the covariant derivatives along the inward-pointing normal to the boundary.

Boundary conditions of the form (1.4) were first considered, from the point of view of pure mathematics, by Gilkey and Smith [2], and have been the object of increasing interest in the analysis of heat-kernel asymptotics [3-7], string theory [8, 9] and Euclidean quantum gravity [10-14]. On the other hand, it is precisely the attempt of applying such a framework to the one-loop semiclassical approximation for the quantized gravitational field which has led to the discovery of a serious technical problem, i.e. the impossibility of achieving a strongly elliptic boundary value problem $[1,15]$. This is a mathematical condition on the uniqueness of the solution of the eigenvalue equation for the leading symbol $\sigma_{L}(P)$ of the gauge-field operator $P$ when restricted to the boundary [1], subject to a decay condition at infinite geodesic distance from the boundary and to the boundary conditions of the problem. If strong ellipticity fails to hold, the leading symbol $\sigma_{L}(P)$ is no longer invertible, and the functional trace of the heat operator acquires a non-integrable part as one approaches the boundary along the inward geodesic flow. The $L^{2}$-trace of $\mathrm{e}^{-t P}$ has then an asymptotic expansion, as $t \rightarrow 0^{+}$, which contains not only the standard interior and boundary terms [16], but also non-integrable contributions [1]. This makes it impossible to use heat-kernel methods (only) to interpret the ultraviolet divergences of the corresponding one-loop quantum theory.

In [17] it has been therefore suggested to look at boundary conditions in Euclidean quantum gravity from the point of view of pseudo-differential boundary value problems. Indeed, not only has the functional calculus of pseudo-differential boundary problems [18] reached a status of high rigour, with several interesting results of general nature, but relevant physical applications are already available in lower-dimensional theories [19]. In the case of gravitational perturbations, the work in [17] has added to the local boundary operator in Eq. (1.4) a non-local boundary operator with kernel $W_{a b}{ }^{c d}\left(x, x^{\prime}\right)$, and special assumptions on the components of $W$ have been made to simplify the resulting calculations. Here we follow instead a more general path because we would like it to understand how far 
can one go in allowing for non-local boundary data. For this purpose, section 2 considers non-local boundary conditions for any bosonic gauge-field theory. Section 3 studies the invariance properties of the boundary operator with the associated consistency conditions. An alternative set of non-local boundary conditions in Euclidean quantum gravity are proposed and studied in section 4, and their counterpart in Euclidean Maxwell theory is derived in section 5. Concluding remarks are presented in section 6, and relevant details are described in the appendix.

\section{A new set of non-local boundary conditions}

For bosonic field theories, non-local boundary conditions add an integral operator to the projectors, endomorphisms and first-order differential operators occurring in the purely local theory (see Eq. (1.4)). For example, as we already said in [17] following [18], in population theory one studies the equation

$$
u(0)-\int_{0}^{\infty} u(t) f(t) d t=0 .
$$

We here consider a pseudo-differential boundary operator defined by a kernel [16], so that Eq. (1.4) is replaced by the boundary condition

$$
\left(\begin{array}{cc}
\Pi+\alpha & \beta \\
\Lambda+\gamma & I-\Pi+\delta
\end{array}\right)\left(\begin{array}{c}
{[\varphi]_{\partial M}} \\
{\left[\varphi_{; N}\right]_{\partial M}}
\end{array}\right)=0
$$

In Eq. (2.2) $\alpha, \beta, \gamma$ and $\delta$ should be viewed as integral operators with kernels $A, B, C$ and $D$, respectively. In the case of metric perturbations $h_{a b}$, their action is taken to be

$$
\begin{aligned}
(\alpha h)_{a b}(x) & \equiv \int_{M} A_{a b}^{p q}\left(x, x-x^{\prime}\right) h_{p q}\left(x^{\prime}\right) d V^{\prime} \\
(\beta h)_{a b}(x) & \equiv \int_{M} B_{a b}^{p q}\left(x, x-x^{\prime}\right) h_{p q}\left(x^{\prime}\right) d V^{\prime} \\
(\gamma h)_{a b}(x) & \equiv \int_{M} C_{a b}^{p q}\left(x, x-x^{\prime}\right) h_{p q}\left(x^{\prime}\right) d V^{\prime}
\end{aligned}
$$




$$
(\delta h)_{a b}(x) \equiv \int_{M} D_{a b}^{p q}\left(x, x-x^{\prime}\right) h_{p q}\left(x^{\prime}\right) d V^{\prime}
$$

where $d V^{\prime}$ is the integration measure over $M$, i.e.

$$
d V^{\prime} \equiv \sqrt{\operatorname{det} g\left(x^{\prime}\right)} d x^{\prime 1} \ldots d x^{\prime m}
$$

The form of the integrands in (2.3)-(2.6) is consistent with our assumption of dealing with a pseudo-differential boundary operator. This is a technical point which is described in detail in the appendix. With the notation defined therein, we say that $A, B, C$ and $D$ are taken to be pseudo-homogeneous kernels. Note that Eq. (2.2) requires taking the restriction to the boundary of what is obtained from (2.3)-(2.6), e.g. (cf [17])

$$
\left[(\alpha h)_{a b}(x)\right]_{\partial M}=\left[\int_{M} A_{a b}^{p q}\left(x, x-x^{\prime}\right) h_{p q}\left(x^{\prime}\right) d V^{\prime}\right]_{\partial M} .
$$

\section{Ghost boundary operator and consistency conditions}

Suppose that our bosonic gauge fields are subject to the infinitesimal gauge transformations (1.2), where the form of the generators $R$ remains, for the time being, not specified. If both $\varphi$ and ${ }^{\varepsilon} \varphi$ satisfy the boundary conditions (2.2), we say that such boundary conditions are preserved under the action of $R$. The resulting boundary conditions on gauge functions are

$$
\begin{gathered}
{\left[(\Pi+\alpha) R \varepsilon+\beta(R \varepsilon)_{; N}\right]_{\partial M}=0} \\
{\left[(\Lambda+\gamma) R \varepsilon+(I-\Pi+\delta)(R \varepsilon)_{; N}\right]_{\partial M}=0 .}
\end{gathered}
$$

Equations (3.1) and (3.2) should provide boundary conditions on the ghost while avoiding an over-determined boundary value problem. For this purpose, assuming that $\Pi+\alpha$ has an inverse, we derive from (3.1) that

$$
[R \varepsilon]_{\partial M}=-\left[(\Pi+\alpha)^{-1} \beta(R \varepsilon)_{; N}\right]_{\partial M} .
$$


The insertion of such a formula into Eq. (3.2) leads to

$$
\left[\left(I-\Pi+\delta-(\Lambda+\gamma)(\Pi+\alpha)^{-1} \beta\right)(R \varepsilon)_{; N}\right]_{\partial M}=0
$$

The condition (3.4) should hold for all $\varepsilon$, to avoid having too many boundary conditions after having imposed Eq. (3.3). Hence it becomes an operator equation which restricts the form of the boundary operator, i.e.

$$
I-\Pi+\delta-(\Lambda+\gamma)(\Pi+\alpha)^{-1} \beta=0
$$

It should be stressed that this equation can only make sense with non-local boundary conditions, since otherwise $\Pi+\alpha$ would reduce to $\Pi$, i.e. a projector for which no inverse exists (the only map satisfying the properties $T^{2}=T$ and $T T^{-1}=T^{-1} T=I$ is the identity $I$ ).

By contrast, if $\beta$ is invertible while $\Pi+\alpha$ cannot be inverted, Eq. (3.1) can be cast in the form

$$
\left[(R \varepsilon)_{; N}\right]_{\partial M}=-\left[\beta^{-1}(\Pi+\alpha) R \varepsilon\right]_{\partial M}
$$

This implies, upon insertion into Eq. (3.2), the consistency condition

$$
\left[\left((\Lambda+\gamma)-(I-\Pi+\delta) \beta^{-1}(\Pi+\alpha)\right)(R \varepsilon)\right]_{\partial M}=0
$$

Since Eq. (3.7) should hold for all $\varepsilon$, it leads to an operator equation that restricts the admissible form of the boundary operator, i.e. (cf (3.5))

$$
\Lambda+\gamma-(I-\Pi+\delta) \beta^{-1}(\Pi+\alpha)=0
$$

For example, the form (3.6) of the ghost boundary conditions should be used when both $\Pi$ and $\Pi+\alpha$ are projection operators, so that the condition

$$
(\Pi+\alpha)^{2}=\Pi+\alpha
$$


implies that

$$
\alpha^{2}+\Pi \alpha+\alpha \Pi=\alpha
$$

The present analysis of consistency conditions for ghost boundary conditions should be supplemented by the general equation which ensures that non-trivial solutions of the system (3.1) and (3.2) exist. This is the operator equation

$$
(\Pi+\alpha)(I-\Pi+\delta)-\beta(\Lambda+\gamma)=0 .
$$

For example, when $\alpha, \beta, \gamma$ and $\delta$ are set to zero, one recovers the identity $\Pi(I-\Pi)=0$ which is satisfied by the boundary operator for local boundary conditions in Eq. (1.4), since $\Pi$ is a projector. Equation (3.11) leads to

$$
\alpha(I+\delta)-\beta \gamma+\Pi \delta-\alpha \Pi-\beta \Lambda=0 .
$$

If we now use the consistency condition (3.5), we can express $I+\delta$ in the form

$$
I+\delta=\Pi+(\Lambda+\gamma)(\Pi+\alpha)^{-1} \beta
$$

and its insertion into Eq. (3.12) yields

$$
\alpha(\Lambda+\gamma)(\Pi+\alpha)^{-1} \beta-\beta(\Lambda+\gamma)+\Pi \delta=0 .
$$

On the other hand, if we use Eq. (3.12) and the consistency condition (3.8), we find from (3.12) that

$$
\Lambda+\gamma=\beta^{-1}[\alpha(I+\delta)+\Pi \delta-\alpha \Pi]
$$

whilst Eq. (3.8) yields

$$
\Lambda+\gamma=(I-\Pi+\delta) \beta^{-1}(\Pi+\alpha) .
$$

The right-hand sides of (3.15) and (3.16) should therefore coincide, which implies (upon application of $\beta$ from the left to both of them)

$$
\alpha(I+\delta)+\Pi \delta-\alpha \Pi=\beta(I-\Pi+\delta) \beta^{-1}(\Pi+\alpha) .
$$




\section{Euclidean quantum gravity}

In Euclidean quantum gravity, the request of invariance under infinitesimal diffeomorphisms of homogeneous Dirichlet conditions on spatial components of metric perturbations leads to homogeneous Dirichlet conditions on the whole ghost 1-form for all boundaries which are not totally geodesic [12] (a boundary is said to be totally geodesic when its extrinsic curvature tensor vanishes). At that stage, the vanishing of the gauge-averaging functional $\Phi_{a}(h)$ at the boundary is imposed to ensure that this remaining set of boundary conditions on metric perturbations leads again to homogeneous Dirichlet conditions on the ghost (with the exception of zero-modes) [1, 10-15]. The difference between the present paper and the scheme considered in $[1,10-15]$ lies, however, in the use of non-local boundary operators (see also [20]).

We therefore assume that $\Phi_{a}(h)$ consists of the de Donder term (which has the advantage of leading to an operator of Laplace type on metric perturbations with purely local boundary conditions) plus a pseudo-differential part with pseudo-homogeneous kernel, i.e. (here $\left.\hat{h} \equiv g^{c d} h_{c d}\right)$

$$
\Phi_{a}(h) \equiv \nabla^{b}\left(h_{a b}-\frac{1}{2} g_{a b} \hat{h}\right)+(\zeta h)_{a}
$$

where

$$
(\zeta h)_{a}(x) \equiv \int_{M} \zeta_{a}^{c d}\left(x, x-x^{\prime}\right) h_{c d}\left(x^{\prime}\right) d V^{\prime} .
$$

As in [1], $\nabla$ is the connection on the bundle of symmetric rank-2 tensor fields over $M$, $g$ is the background metric, and $d V^{\prime}$ is the integration measure over $M$ used already in (2.3)-(2.6). Moreover, $\zeta_{a}^{c d}\left(x, x-x^{\prime}\right)$ is taken to be a pseudo-homogeneous kernel on the curved $m$-dimensional Riemannian background $(M, g)$. By virtue of the assumption (4.2), the standard rule for the evaluation of the ghost operator $\mathcal{F}_{a}^{b}$ yields now a non-trivial result, because (see (1.2))

$$
\Phi_{a}(h)-\Phi_{a}\left({ }^{\varepsilon} h\right)=\mathcal{F}_{a}^{b} \varepsilon_{b}
$$

where (here $\square \equiv g^{a b} \nabla_{a} \nabla_{b}$ )

$$
\mathcal{F}_{a}^{b} \equiv-\delta_{a}^{b} \square-R_{a}^{b}+\widetilde{\mathcal{F}}_{a}^{b}
$$


$\widetilde{\mathcal{F}}_{a}^{b}$ being the pseudo-differential operator defined by

$$
\widetilde{\mathcal{F}}_{a}^{b} \varepsilon_{b}(x) \equiv-\int_{M} \zeta_{a}^{c d}\left(x, x-x^{\prime}\right) \nabla_{(c} \varepsilon_{d)}\left(x^{\prime}\right) d V^{\prime}
$$

The gauge-field operator $P_{a b}{ }^{c d}$ on metric perturbations is obtained by expanding to quadratic order in $h_{a b}$ the Euclidean Einstein-Hilbert action and adding the integral over $M$ of $\frac{\Phi_{a}(h) \Phi^{a}(h)}{2 \alpha}$. On setting $\alpha=1$ for simplicity, one finds that

$$
P_{a b}{ }^{c d}=G_{a b}{ }^{c d}+U_{a b}{ }^{c d}+V_{a b}{ }^{c d} .
$$

With our notation, $G_{a b}{ }^{c d}$ is the operator of Laplace type given by [13]

$$
\begin{aligned}
G_{a b}^{c d} & \equiv E_{a b}^{c d}(-\square+R)-2 E_{a b}^{q f} R_{q p f}^{c} g^{d p} \\
& -E_{a b}^{p d} R_{p}^{c}-E_{a b}{ }^{c p} R_{p}^{d}
\end{aligned}
$$

where $E^{a b c d}$ is the DeWitt supermetric (i.e. the metric on the vector bundle of symmetric rank-2 tensor fields over $M$ )

$$
E^{a b c d} \equiv \frac{1}{2}\left(g^{a c} g^{b d}+g^{a d} g^{b c}-g^{a b} g^{c d}\right)
$$

Moreover, $U_{a b}{ }^{c d}$ and $V_{a b}{ }^{c d}$ are pseudo-differential operators resulting from $\Phi_{a}(h)(\zeta h)^{a}$ and $\frac{(\zeta h)_{a}(\zeta h)^{a}}{2}$ respectively, in the expression of the gauge-averaging term $\frac{\Phi_{a}(h) \Phi^{a}(h)}{2}$. To work out the former, we use the identity

$$
\begin{aligned}
& \nabla^{b}\left[\left(h_{a b}-\frac{1}{2} g_{a b} \hat{h}\right) \int_{M} \zeta^{a c d}\left(x, x-x^{\prime}\right) h_{c d}\left(x^{\prime}\right) d V^{\prime}\right] \\
& \equiv \nabla^{b} T_{b} \\
& =\left[\nabla^{b}\left(h_{a b}-\frac{1}{2} g_{a b} \hat{h}\right)\right] \int_{M} \zeta^{a c d}\left(x, x-x^{\prime}\right) h_{c d}\left(x^{\prime}\right) d V^{\prime} \\
& +\left(h_{a b}-\frac{1}{2} g_{a b} \hat{h}\right) \nabla^{b} \int_{M} \zeta^{a c d}\left(x, x-x^{\prime}\right) h_{c d}\left(x^{\prime}\right) d V^{\prime} .
\end{aligned}
$$


We therefore define

$$
T_{b} \equiv\left(h_{a b}-\frac{1}{2} g_{a b} \hat{h}\right)(\zeta h)^{a}
$$

and add to the action a boundary term equal to $\left(d \Sigma^{\prime}\right.$ being the integration measure over $\partial M)$

$$
-\int_{\partial M} N^{b} T_{b} d \Sigma^{\prime}
$$

to find that the action of $U_{a b}{ }^{c d}$ is defined by

$$
U_{a b}^{c d} h_{c d}(x) \equiv-2 \nabla^{r} E_{r s a b} \int_{M} \zeta^{s c d}\left(x, x-x^{\prime}\right) h_{c d}\left(x^{\prime}\right) d V^{\prime}
$$

Furthermore, the definition (4.2) implies that $V_{a b}{ }^{c d}$ is a pseudo-differential operator whose action is defined by

$$
\begin{aligned}
& h^{a b} V_{a b}{ }^{c d} h_{c d}(x) \\
& \equiv \int_{M^{2}} h^{a b}\left(x^{\prime}\right) \zeta_{p a b}\left(x, x-x^{\prime}\right) \zeta^{p c d}\left(x, x-x^{\prime \prime}\right) h_{c d}\left(x^{\prime \prime}\right) d V^{\prime} d V^{\prime \prime} .
\end{aligned}
$$

It should be stressed that the scheme considered in the present section differs substantially from the analysis of sections 2 and 3, because we have imposed the following boundary conditions on metric perturbations:

$$
\begin{gathered}
{\left[h_{i j}\right]_{\partial M}=0} \\
{\left[\Phi_{a}(h)\right]_{\partial M}=\left[\nabla^{b}\left(h_{a b}-\frac{1}{2} g_{a b} \hat{h}\right)+(\zeta h)_{a}\right]_{\partial M}=0}
\end{gathered}
$$

Both (4.13) and (4.14) are invariant under infinitesimal diffeomorphisms if the whole ghost 1-form vanishes at the boundary, i.e.

$$
\left[\varepsilon_{a}\right]_{\partial M}=0
$$

Thus, we have proposed the general equations for an approach to Euclidean quantum gravity where both metric perturbations and ghost fields are ruled by pseudo-differential 
operators, while the boundary conditions have a Dirichlet and a pseudo-differential sector (and are pure Dirichlet for the ghost). As we know from the general analysis in [17] and [18], the adjoint of the ghost operator will instead be a differential operator, but subject to non-local boundary conditions, to ensure self-adjointness.

\section{Quantized Maxwell theory}

It may be now helpful to show how the scheme of section 4 can be applied to a simpler field theory, i.e. Euclidean Maxwell theory. For this purpose, we consider a gauge-averaging functional $\Phi(A)$ given by the Lorenz term (i.e. the counterpart of the de Donder term for gravity) plus a pseudo-differential contribution $Q(A)$ with pseudo-homogeneous kernel $Q^{b}\left(x, x-x^{\prime}\right)$, i.e.

$$
\Phi(A) \equiv \nabla^{b} A_{b}+Q(A)
$$

where

$$
Q(A)(x) \equiv \int_{M} Q^{b}\left(x, x-x^{\prime}\right) A_{b}\left(x^{\prime}\right) d V^{\prime} .
$$

With our notation, $\nabla$ is now the connection on the vector bundle $W$ of 1 -forms $A_{b} d x^{b}$ over $M$. The metric on $W$ coincides with the metric $g$ of $M$, and infinitesimal gauge transformations read

$$
{ }^{\varepsilon} A_{b} \equiv A_{b}+\nabla_{b} \varepsilon .
$$

By virtue of the hypothesis (5.1), the ghost operator $\mathcal{F}$ is found from the equation

$$
\Phi(A)-\Phi\left({ }^{\varepsilon} A\right)=\mathcal{F} \varepsilon
$$

where

$$
\mathcal{F} \equiv-\square+\widetilde{\mathcal{F}}
$$

$\widetilde{\mathcal{F}}$ being the pseudo-differential operator defined by

$$
\widetilde{\mathcal{F}} \varepsilon(x) \equiv-\int_{M} Q^{b}\left(x, x-x^{\prime}\right)\left(\nabla_{b} \varepsilon\right)\left(x^{\prime}\right) d V^{\prime}
$$


The gauge-field operator $P_{a}^{b}$ on perturbations of the potential (the background value of $A$ is here taken to vanish) is obtained by expanding to quadratic order in $A$ the Euclidean Maxwell action and adding the integral over $M$ of $\frac{[\Phi(A)]^{2}}{2 \alpha}$. On setting again $\alpha=1$ for simplicity as in section 4 , one finds that

$$
P_{a}^{b}=G_{a}^{b}+U_{a}^{b}+V_{a}^{b}
$$

We denote by $G_{a}^{b}$ the operator of Laplace type

$$
G_{a}^{b} \equiv-\delta_{a}^{b} \square+R_{a}^{b}
$$

Furthermore, $U_{a}^{b}$ and $V_{a}^{b}$ are pseudo-differential operators. The former is obtained by using the identity

$$
\nabla^{b}\left(A_{b} Q(A)\right)=\left(\nabla^{b} A_{b}\right) Q(A)+A_{b} \nabla^{b} Q(A)
$$

Thus, on defining

$$
\widetilde{T}_{b} \equiv A_{b} Q(A)
$$

we add to the action integral for the 1-loop quantum theory a boundary term equal to

$$
-\int_{\partial M} N^{b} \widetilde{T}_{b} d \Sigma^{\prime}
$$

to find that the action of $U_{a}^{b}$ is defined by

$$
U_{a}^{b} A_{b}(x) \equiv-2 \nabla_{a} \int_{M} Q^{b}\left(x, x-x^{\prime}\right) A_{b}\left(x^{\prime}\right) d V^{\prime} .
$$

Last, the operator $V_{a}^{b}$ results from $Q^{2}(A)$ in $[\Phi(A)]^{2}$, and is such that

$$
\begin{aligned}
& A^{a} V_{a}^{b} A_{b}(x) \\
& \equiv \int_{M^{2}} A^{a}\left(x^{\prime}\right) Q_{a}\left(x, x-x^{\prime}\right) Q^{b}\left(x, x-x^{\prime \prime}\right) A_{b}\left(x^{\prime \prime}\right) d V^{\prime} d V^{\prime \prime} .
\end{aligned}
$$

The gauge-invariant boundary conditions which represent the electromagnetic counterpart of Eqs. (4.13) and (4.14) for gravity are (cf [13])

$$
\left[\left(\delta_{a}^{b}-N_{a} N^{b}\right) A_{b}\right]_{\partial M}=0
$$




$$
[\Phi(A)]_{\partial M}=\left[\nabla^{b} A_{b}+Q(A)\right]_{\partial M}
$$

where the square bracket on the left-hand side of Eq. (5.13) represents the tangential components of the potential. Both Eq. (5.13) and (5.14) are preserved under the action of gauge transformations (5.3) provided that the ghost field vanishes at the boundary:

$$
[\varepsilon]_{\partial M}=0
$$

where Eq. (5.15) should also hold for the anti-ghost [13, 22].

\section{Concluding remarks}

The theory of elliptic operators on compact Riemannian manifolds $(M, g)$ with or without boundary is a fascinating topic which has led to several deep developments in mathematics and theoretical physics [21]. There is, first, the Dirac operator obtained by composing Clifford multiplication with covariant differentiation [21], i.e.

$$
\mathcal{D} \equiv \gamma^{a} \nabla_{a}
$$

whose symbol generates all elliptic symbols on a compact Riemannian manifold, so that this operator may be viewed as the most fundamental among all elliptic operators (and also very well suited to studying topological properties of vector bundles over $M$ ).

The Laplace operator or, more generally, operators of Laplace type

$$
\begin{gathered}
P: C^{\infty}(V, M) \rightarrow C^{\infty}(V, M) \\
P \equiv-g^{a b} \nabla_{a} \nabla_{b}-E
\end{gathered}
$$

occurs whenever one tries to quantize bosonic gauge theories in linear covariant gauges via path-integral methods [22]. Its leading symbol is scalar $[1,16]$.

The next step is the consideration of non-minimal operators, whose leading symbol is

not of scalar type [16]. They are relevant for the quantization of bosonic gauge theories in arbitrary gauges. An example is provided by the gauge-field operator when the Lorenz 
gauge-averaging term is used for Euclidean Maxwell theory with arbitrary gauge parameter $\alpha$, i.e.

$$
P_{a}^{b} \equiv-\delta_{a}^{b} \square+\left(1-\frac{1}{\alpha}\right) \nabla_{a} \nabla^{b}+R_{a}^{b}
$$

A further step is given by the analysis of conformally covariant operators $Q$. Their consideration is suggested by the behaviour under conformal rescalings

$$
g_{a b} \rightarrow \mathrm{e}^{2 \Omega} g_{a b}
$$

of the metric, since they are then found to transform according to the rule

$$
Q(\Omega)=\mathrm{e}^{-(m+4) \Omega / 2} Q(\Omega=0) \mathrm{e}^{(m-4) \Omega / 2} .
$$

In other words, conformally covariant operators occur whenever conformal symmetries play a key role in the process of understanding some features of a field theory (e.g. supplementary conditions which are conformally invariant $[23,24])$.

Still, all the above operators are only particular cases of the general family of pseudodifferential operators $[16,18,25]$. While differential operators $D$ are local in that the equation $f=0$ implies $D f=0$ as well, this is no longer true for pseudo-differential operators, because their construction in $\mathbf{R}^{m}$ involves the Fourier transform, which smears out the support [16]. Moreover, their definition can be extended to Riemannian manifolds in a coordinate-free way [16]. A careful analysis of the role of pseudo-differential operators in Euclidean quantum gravity is therefore quite important, because all what has been achieved so far $[13,26]$ is only a particular case of a more general framework which is, to a large extent, unexplored in the literature on quantum gravity [17].

In our paper, we have first considered non-local boundary conditions in the form (2.2), with the action of $\alpha, \beta, \gamma$ and $\delta$ defined by polyhomogeneous kernels as in (2.3)-(2.6). The preservation of such boundary conditions under infinitesimal gauge transformations leads to the ghost boundary conditions (3.1) and (3.2). Actually, Eq. (3.1) has been required to specify completely the behaviour of ghost fields at the boundary. Equation (3.2) becomes then a consistency condition which restricts the admissible forms of non-local boundary 
operators (see (3.5) and (3.8)). On the other hand, non-trivial solutions of the system given by (3.1) and (3.2) exist if and only if Eq. (3.11) holds. Its compatibility with Eq. (3.5) leads to Eq. (3.14), whereas its compatibility with Eq. (3.8) yields Eq. (3.17).

In Euclidean quantum gravity, an alternative scheme has been built, with gaugeaveraging functional given by (4.1) and (4.2), ghost operator in the form (4.4) and (4.5), operator on metric perturbations described by (4.6), (4.7), (4.11) and (4.12). Interestingly, non-local boundary conditions, jointly with the request of their complete gauge invariance, are sufficient to lead to gauge-field and ghost operators of pseudo-differential nature. The resulting ghost boundary conditions are homogeneous Dirichlet as in (4.15), and hence differ from (3.1) and (3.2).

Section 5 has shown in detail how the formalism of section 4 can also be applied to the 1-loop semiclassical theory of the free Maxwell field in vacuum. Once more, gaugeinvariant boundary conditions on the gauge field given by a direct sum of a Dirichlet and a pseudo-differential sector are sufficient to lead to gauge-field and ghost operators of pseudo-differential nature.

It now remains to be seen which one, among the schemes of sections 3 and 4 , is best suited to satisfy the requirement of strong ellipticity of the pseudo-differential boundary value problem (see definitions and theorems in section 1.7 of [18]). Moreover, kernels of distributional nature, rather than the polyhomogeneous kernels advocated in our paper, might be studied as well, generalizing to curved backgrounds the analysis in [27]. At the present stage, it is unclear whether both families of kernels are needed in quantum gravity, or whether one of the two is more fundamental. Last, but not least, it appears desirable to investigate the role of non-local boundary conditions in quantum cosmology [13, 26, 28], with application to 1-loop semiclassical effects and large-scale structure of the universe.

\section{Acknowledgments}

This work has been partially supported by PRIN97 'Sintesi'. The author is much indebted to Ivan Avramidi and Alexander Kamenshchik for years of joint work on the boundary conditions in Euclidean quantum gravity. He would like to dedicate the present paper to Michela Foa. 


\section{Appendix}

In [17] we have given some basic definitions about pseudo-differential operators and their symbols. In the present paper, however, we are interested in kernels of pseudo-differential operators, and hence we need a more advanced treatment. For this purpose, we follow chapter III of [25]. The basic concepts and results we need are as follows.

Definition 1. Let $\omega$ be a complex number such that $\omega \neq 0,1,2, \ldots$, and $\operatorname{Re}(\omega)>-\nu$. A function $f$ is a $C^{\infty}$ pseudo-homogeneous function of degree $\omega$, denoted by $\psi h f_{\omega}$, if and only if outside the origin $f$ is a $C^{\infty}$ function homogeneous of degree $\omega$ :

$$
f(t x)=t^{\omega} f(x) .
$$

Moreover, if $\omega=0,1,2, \ldots$, then $f$ is a $\psi h f_{\omega}$ if and only if

$$
f(x)=P(x) \log |x|+g(x)
$$

where $P$ is a polynomial of degree $\omega$, and $g$ is a $C^{\infty}$ function homogeneous of degree $\omega$, for which

$$
\int_{|x|=1} x^{\alpha} g(x)=0 \quad|\alpha|=\omega .
$$

Definition 2. Let $U \subset \mathbf{R}^{\nu}$ be open, and let $K(x, x-y)$ be defined for $x, y$ in $U$, with $x \neq y$. Let $\operatorname{Re}(\omega)>-\nu$. Then $K$ is a pseudo-homogeneous kernel of degree $\omega$, denoted by $\psi h k_{\omega}$, if and only if there exist functions $K_{\omega+j}(x, z)$, of class $C^{\infty}$ for $z \neq 0$ and pseudo-homogeneous in $z$ of degree $\omega+j$, such that

$$
K(x, x-y)-\sum_{j<J} K_{\omega+j}(x, x-y)
$$

is of class $C^{k}$ for $k<\operatorname{Re}(\omega)+J$.

Theorem 1. Let $\operatorname{Re}(\omega)<0$. Then

$$
A: C_{c}^{\infty}(U) \rightarrow C^{\infty}(U)
$$


is a pseudo-differential operator of degree $\omega$ if and only if

$$
A f(x)=\int_{U} K(x, x-y) f(y) d y
$$

where $K$ is a pseudo-homogeneous kernel of degree $-\omega-\nu$.

A theory with $\operatorname{Re}(\omega) \geq 0$ might also be developed, leading to distributional kernels, as is shown in [27]. Two general principles underlie Theorem 1:

(i) If the kernel $K$ is homogeneous of degree $s$, then its Fourier transform $\hat{K}$ is homogeneous of degree $-s-\nu$. One can indeed write

$$
\hat{K}(t \xi)=\int \mathrm{e}^{i x t \xi} K(x) d x
$$

and upon setting $x t=y$ one finds

$$
\hat{K}(t \xi)=\int \mathrm{e}^{i y \xi} K\left(y t^{-1}\right) t^{-\nu} d y=t^{-s-\nu} \hat{K}(\xi)
$$

which proves our statement. However, the integral (A.5) is not absolutely convergent for any value of $s$, and not even conditionally convergent for most values of $s$.

(ii) The behaviour of $\hat{K}$ near $\infty$ is closely related to the behaviour of $K$ near 0 . This property can be exploited to circumvent the convergence problem.

Interestingly, one can pass from a pseudo-homogeneous kernel $K_{s}$ to a symbol $a_{-s-\nu}$; one recovers $a_{-s-\nu}$ indirectly from the function $\xi^{\alpha} a_{-s-\nu}$ for sufficiently large $\alpha$.

All the above definitions and properties hold for pseudo-differential operators on $\mathbf{R}^{m}$. On extending the formalism to generic $m$-dimensional Riemannian manifolds $(M, g)$, it

remains to be proved in a rigorous way that it is sufficient to replace $d y \equiv d y^{1} \ldots d y^{m}$ in (A.4) by the integration measure $\sqrt{\operatorname{det} g(y)} d y^{1} \ldots d y^{m}$ (see section 1.3 of [16]).

\section{References}

[1] Avramidi I G and Esposito G 1999 Commun. Math. Phys. 200495 
[2] Gilkey P B and Smith L 1983 J. Diff. Geom. 18393

[3] McAvity D M and Osborn H 1991 Class. Quantum Grav. 81445

[4] Dowker J S and Kirsten K 1997 Class. Quantum Grav. 14 L169

[5] Avramidi I G and Esposito G 1998 Class. Quantum Grav. 15281

[6] Elizalde E and Vassilevich D V 1999 Class. Quantum Grav. 16813

[7] Dowker J S and Kirsten K 1999 Class. Quantum Grav. 161917

[8] Abouelsaood A, Callan C G, Nappi C R and Yost S A 1987 Nucl. Phys. B 280599

[9] Callan C G, Lovelace C, Nappi C R and Yost S A 1987 Nucl. Phys. B 288525

[10] Barvinsky A O 1987 Phys. Lett. 195B 344

[11] Esposito G, Kamenshchik A Yu, Mishakov I V and Pollifrone G 1995 Phys. Rev. D 523457

[12] Avramidi I G, Esposito G and Kamenshchik A Yu 1996 Class. Quantum Grav. 13 2361

[13] Esposito G, Kamenshchik A Yu and Pollifrone G 1997 Euclidean Quantum Gravity on Manifolds with Boundary (Fundamental Theories of Physics 85) (Dordrecht: Kluwer)

[14] Moss I G and Silva P J 1997 Phys. Rev. D 551072

[15] Avramidi I G and Esposito G 1998 Class. Quantum Grav. 151141

[16] Gilkey P B 1995 Invariance Theory, the Heat Equation and the Atiyah-Singer Index Theorem (Boca Raton, FL: Chemical Rubber Company)

[17] Esposito G 1999 Class. Quantum Grav. 161113

[18] Grubb G 1996 Functional Calculus of Pseudodifferential Boundary Problems (Progress of Mathematics 65) (Boston: Birkhäuser)

[19] Schröder M 1989 Rep. Math. Phys. 27259

[20] Marachevsky V N and Vassilevich D V 1996 Class. Quantum Grav. 13645

[21] Esposito G 1998 Dirac Operators and Spectral Geometry (Cambridge Lecture Notes in Physics vol 12) (Cambridge: Cambridge University Press)

[22] DeWitt B S in Relativity, Groups and Topology II eds B S DeWitt and R Stora (Amsterdam: North-Holland)

[23] Eastwood M and Singer I M 1985 Phys. Lett. A 10773

[24] Esposito G 1997 Phys. Rev. D 562442 
[25] Seeley R T 1969 Topics in pseudo-differential operators, C.I.M.E., in Conf. on PseudoDifferential Operators, ed. L. Nirenberg (Roma: Edizioni Cremonese)

[26] Gibbons G W and Hawking S W 1993 Euclidean Quantum Gravity (Singapore: World Scientific)

[27] Kree P Les noyaux des operateurs pseudo-differentials, Publications du séminaire de mathematiques de l'Université de Bari

[28] Hartle J B and Hawking S W 1983 Phys. Rev. D 282960 